Pacific Journal of Mathematics

TS WITH ZERO-DIMENSIONAL KERNELS

al Eugene roland and John M. Mark 


\title{
SETS WITH ZERO-DIMENSIONAL KERNELS
}

\author{
N. E. Foland AND J. M. MARR
}

\begin{abstract}
F. A. Valentine in his book ([1], p. 177, Problem 6.5) suggests that a sufficient condition for a nonempty compact and connected subset $S$ of $E^{2}$ to have a kernel consisting of a single point is that each triple of points of $S$ can see via $S$ a unique point of $S$. The authors show that this condition is sufficient if $S$ is any subset of a topological linear space which contains a noncollinear triple.
\end{abstract}

Let $S$ be a subset of a linear space. A point $p$ is said to be in the kernel of $S$ if $p$ can be joined to each point of $S$ by a closed line segment that lies in S. F. A. Valentine ([1], p. 164, Problem 1.1) has posed the problem of determining conditions under which the kernel of $S$ will be 0-dimensional, that is, will consist of a single point. $\mathrm{He}$ suggests ([1], p. 177, Problem 6.5) that if $S$ is a compact and connected subset of the euclidean plane, then a sufficient condition for the kernel of $S$ to be a single point is that each noncollinear triple of points of $S$ be able to see a unique point of $S$ via $S$, where this unique point depends on the triple chosen. In this paper we prove that this condition on the noncollinear triples of $S$ implies that the kernel of $S$ is 0 -dimensional if $S$ is any subset of a topological linear space containing a noncollinear triple. Thus we establish a stronger result than that suggested by Valentine. The result is secured by first establishing, in the form of lemmas, several properties of such a set $S$ that culminate in the main result. The major property established, namely, that such a set $S$ cannot contain a closed polygonal path made up of four line segments, is obtained in Lemmas 2 and 3 . The notation and terminology is that used in [1].

Sufficient conditions for a set $S$ to have a one point kernel. In the sequel $S$ will denote a subset of a topological linear space $L$ over the real field $\mathscr{R}$ with the property that if $x, y, z$ are noncollinear points of $S$, then there exists a unique point $p$ of $S$ such that the three segments $x p, y p, z p$ all lie in $S$. (Here the point $p$ may be one of the points $x, y$ or $z$ in which case one of the segments will be degenerate.) By the kernel $K$ of $S$ we mean the set of all points $p \in S$ with the property that if $x \in S$, then the segment $x p \subset S$.

Lemma 1. If $x, y \in S, x \neq y, x y \subset S$ and $z \in S$ such that $x, y, z$ are noncollinear, then the unique point $p$ of $S$ with the property that $x p, y p$, zp all lie in $S$ is on the line determined by $x$ and $y$. 
Proof. Note first that $S$ can not contain a triangle. (The vertices of a triangle form a noncollinear triple of points and if the triangle lies in $S$, then the point corresponding to the vertices in the assumed condition on noncollinear triples of $S$ may be taken as any one of the vertices, contradicting the uniqueness of this point.) Thus if the point $p$ of the lemma is not on the line determined by $x$ and $y$, then $S$ contains a triangle.

By the dimension of a subset of $L$ we mean the dimension of a flat (also called linear variety) of least dimension that contains the set. A 1-dimensional flat (2-dimensional flat) will be called a line (a plane).

LEMma 2. The set $S$ can not contain a closed polygon consisting of four line segments that lie in a plane.

Proof. In order to show this, suppose the contrary and let $a, b, c, d$ be the vertices of such a quadrilateral $Q$ with sides $a b, b c, c d, d a$ all in $S$. If the quadrilateral $Q$ is the boundary of a nonconvex subset of $L$, then one of the vertices, say $a$, is contained in the interior of the triangle formed by the remaining vertices. Let $t$ be any point on side $a b$ of $Q$ that lies between $a$ and $b$. Since $t, d$, and $c$ are noncollinear points of $S$ and since $c d \subset S$, the point $t$ can be joined by a segment in $S$ to a point $r$ of the line of $c$ and $d$. It follows that either the points $a, d, r, t$ or the points $t, b, c, r$ form the vertices of a quadrilateral that is the boundary of a convex subset of the linear space $L$. Thus we may assume that $Q$ is the boundary of a convex subset of $L$.

Let $i(Q)$ denote the set bounded by $Q$ relative to the plane containing $Q$. We now show that if $Q \subset S$, then $i(Q) \subset S$. This is clearly impossible since $i(Q)$ contains a triangle. Let $t$ be any point of side $a b$ of $Q$ such that $a \neq t \neq b$. Since $S$ contains no triangle and the points $t, c, d$ are noncollinear, the unique point $r_{t}$ of $S$ to which each of the points $t, c, d$ can be joined by a segment in $S$ must be on side $c d$ of $Q$ between $c$ and $d$. Let $p \in i(Q)$ and suppose $p \notin S$. Then for each $t \in a b$, either $p$ is a point of that part of $i(Q)$ that is bounded by $t b, b c, c r_{t}$, and $t r_{t}$ or $p$ is in that part of $i(Q)$ bounded by $a t, t r_{t}$, $r_{t} d$, and $d a$. Let $A$ be the set of all $t \in a b$ such that $p$ is in that part of $i(Q)$ bounded by $t b, b c, c r_{t}, t r_{t}$; and let $B=a b-A$. Then $a \in A$ and $b \in B$. If $u$ and $v$ are distinct points on $a b$, then the segments $u r_{u}$ and $v r_{v}$ do not intersect, where $r_{u}$ and $r_{v}$ denote the unique points on side $c d$ to which $u$ and $v$ can be joined, respectively. Thus if $u \in A$ and $v \in a b$ such that the order, $a, v, u$ holds on $a b$, then $v \in A$. It follows from this and the definition of $A$ and $B$, that either $A$ has a last point or $B$ has a first point in the order from $a$ to $b$. Suppose 
that $s$ is the last point of $A$ in the order from $a$ to $b$. Then $s \neq b$ and $p$ is inside the quadrilateral with sides $s b, b c, c r_{s}$, and $s r_{s}$. Let $w$ be any point of the segment $a b$ between $s$ and $b$. Then $w \in B$ and $p$ is inside the quadrilateral with sides $a w, w r_{w}, d r_{w}$, and $d a$. Thus for each such $w, p$ is inside the quadrilateral with sides $s w, w r_{w}, r_{w} r_{s}$, and $s r_{s}$. Now let the point $w$ converge to the point $s$ along the segment $a b$, then the point $r_{w}$ converges to the point $r_{s}$ on $c d$, for if this is not the case $S$ contains a triangle. This implies that $p \in s r_{s}$ which is contrary to our assumption. If $s$ is the first point of $B$ in the order from $a$ to $b$ the argument is similar. Thus the lemma is proved.

Lemma 3. Let $S$ contain a noncollinear triple. If $x, y \in S$ such that $x y \not \subset S$, then there is a unique point $p \in S$ such that $x p$ and $y p$ both lie in $S$.

Proof. Since the set $S$ is not linear there is at least one point $p \in S$ such that $x p, y p \subset S$. Let $q \in S, \quad p \neq q$, and suppose that $x q, y q \subset S$. By Lemma 2 the four points $x, y, p, q$ do not lie in a plane. Thus the situation is as follows: No one of the four points $x, y, p, q$ is in the plane determined by the other three and exactly four of the six segments determined by these four points, namely, $x p, y p, x q, y q$, lie in $S$. Denote the plane determined by three noncollinear points $a, b, c$ of $L$ by $\pi(a b c)$.

We now show that if $z \in S$ such that $z p \subset S$, then $z \in \pi(x y p)$. Suppose $z p \subset S$ and $z \notin \pi(x y p)$. If $p, q, z$ are noncollinear, then the unique point $r \in S$ to which each can be joined by a segment in $S$ must be on the line determined by $z$ and $p$. Thus the points $x, y$ and $r$ are collinear since each can be joined by a segment in $S$ to the distinct points $p$ and $q$. This implies $r \in \pi(x y p)$ which is impossible since the line determined by $z$ and $p$ has only the point $p$ in common with $\pi(x y p)$. Hence $p, q, z$ are collinear. Since $p, q$, and $z$ are collinear, the points $y, q$, and $z$ are noncollinear. Thus the unique point $r \in S$ to which $y, q, z$ can be joined by a segment in $S$ is on the line determined by $y$ and $q$. This implies that $S$ contains a triangle or a plane quadrilateral since $z \in \pi(y p q)$. Thus if $z \in S$ such that $z p \subset S$, then $z \in \pi(x y p)$. It follows that if $z \in S$ such that $z$ can be joined by a segment in $S$ to one of the points $x, y, p, q$ then $z$ is in the plane determined by the two segments in the set $\{x p, y p, x q, y q\}$ that have this point in common.

Consider now a point $u$ on $x q$ between $x$ and $q$ and a point $v$ on $y q$ between $y$ and $q$ such that the plane $\pi(u v p)$ does not intersect the line of $x$ and $y$. Then there is a point $s \in \pi(x y p)$ to which each of 
the points $u, v, p$ can be joined by segments in $S$. Since $p \in S$ and $p s \subset S, p \in \pi(u v s)$. This is true since we can replace $x, y, p$ in the argument in the preceding paragraph by $u, v, s$. Thus $s$ is on the line of intersection of $\pi(x y p)$ and $\pi(u v p)$. The points $p, s, q$ are noncollinear and hence the point $q$ can be joined by a segment in $S$ to a point $t$ of the line of $s$ and $p$. Since $q t \subset S, t \in \pi(x y q)$. This is impossible since $\pi(x y p)$ and $\pi(x y q)$ have only the line of $x$ and $y$ in common.

This completes the proof of the lemma.

THEOREM. If $S$ contains a noncollinear triple, then the kernel $K$ of $S$ consists of a single point.

Proof. Since $S$ contains a noncollinear triple it contains at least two noncollinear line segments with a common endpoint $p$. We will show that $K=\{p\}$. Let $x$ and $y$ denote the other two endpoints of this two segment path in $S$, and let $q$ be any point of $S$ not on this two segment path. If $x, y, q$ are noncollinear, then the unique point $r$ of $S$ to which each can be joined by a segment in $S$ must be the point $p$ by Lemma 3 . If the points $x, y, q$ are collinear, then the points $x, p, q$ are noncollinear and the points $y, p, q$ are noncollinear. Thus the point $q$ can be joined by a segment in $S$ to a point $r$ on the line of $x$ and $p$. Also $q$ can be joined by a segment in $S$ to a point $t$ of the line of $y$ and $p$. If the points $p, r, t$ are distinct, then we have a contradiction of Lemma 3. If exactly two of the points $p, r, t$ are the same, then $S$ contains a traingle. It follows that $p=r=t$ and $p q \subset S$. Thus the point $p$ is in the kernel $K$ of $S$. It is clear that $K$ can not contain two distinct points. Thus the theorem is proved.

The assumed condition on triples of $S$ is not a necessary condition for $K$ to consist of a single point. This can be seen by considering a triangle together with its interior and with two of its sides extended through the base opposite their intersection.

\section{REFERENCE}

1. F. A. Valentine, Convex sets, McGraw-Hill Series in Higher Mathematics, McGrawHill, Inc., New York, 1964.

Received June 18, 1965.

SOUTHERN ILLINOIS UNIVERSITY

KANSAS State University 


\section{PACIFIC JOURNAL OF MATHEMATICS}

\section{EDITORS}

H. SAMELSON

Stanford University

Stanford, California

J. P. JANS

University of Washington

Seattle, Washington 98105

\section{J. DuGUNDJI}

University of Southern California

Los Angeles, California 90007

RICHARD ARENS

University of California

Los Angeles, California 90024

\section{ASSOCIATE EDITORS}

E. F. BECKENBACH
B. H. NEUMANN

F. WOLF

K. YoSIDA

\section{SUPPORTING INSTITUTIONS}

\author{
UNIVERSITY OF BRITISH COLUMBIA \\ CALIFORNIA INSTITUTE OF TECHNOLOGY \\ UNIVERSITY OF CALIFORNIA \\ MONTANA STATE UNIVERSITY \\ UNIVERSITY OF NEVADA \\ NEW MEXICO STATE UNIVERSITY \\ OREGON STATE UNIVERSITY \\ UNIVERSITY OF OREGON \\ OSAKA UNIVERSITY \\ UNIVERSITY OF SOUTHERN CALIFORNIA
}

\author{
STANFORD UNIVERSITY \\ UNIVERSITY OF TOKYO \\ UNIVERSITY OF UTAH \\ WASHINGTON STATE UNIVERSITY \\ UNIVERSITY OF WASHINGTON \\ AMERICAN MATHEMATICAL SOCIETY \\ CHEVRON RESEARCH CORPORATION \\ TRW SYSTEMS \\ NAVAL ORDNANCE TEST STATION
}

Mathematical papers intended for publication in the Pacific Journal of Mathematics should be typewritten (double spaced). The first paragraph or two must be capable of being used separately as a synopsis of the entire paper. It should not contain references to the bibliography. Manuscripts may be sent to any one of the four editors. All other communications to the editors should be addressed to the managing editor, Richard Arens at the University of California, Los Angeles, California 90024 .

50 reprints per author of each article are furnished free of charge; additional copies may be obtained at cost in multiples of 50 .

The Pacific Journal of Mathematics is published monthly. Effective with Volume 16 the price per volume (3 numbers) is $\$ 8.00$; single issues, $\$ 3.00$. Special price for current issues to individual faculty members of supporting institutions and to individual members of the American Mathematical Society: $\$ 4.00$ per volume; single issues $\$ 1.50$. Back numbers are available.

Subscriptions, orders for back numbers, and changes of address should be sent to Pacific Journal of Mathematics, 103 Highland Boulevard, Berkeley 8, California.

Printed at Kokusai Bunken Insatsusha (International Academic Printing Co., Ltd.), No. 6, 2-chome, Fujimi-cho, Chiyoda-ku, Tokyo, Japan.

\section{PUBLISHED BY PACIFIC JOURNAL OF MATHEMATICS, A NON-PROFIT CORPORATION}

The Supporting Institutions listed above contribute to the cost of publication of this Journal, but they are not owners or publishers and have no responsibility for its content or policies. 


\section{Pacific Journal of Mathematics

Vol. 19, No. $3 \quad$ July, 1966

S. J. Bernau, The spectral theorem for unbounded normal operators .......

Lu-san Chen, Asymptotic behavior of solutions of parabolic equations of

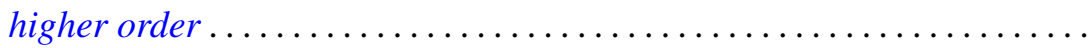

Lawrence William Conlon, An application of the Bott suspension map to the

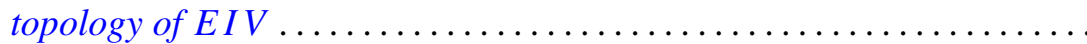

Neal Eugene Foland and John M. Marr, Sets with zero-dimensional kernels .........................................

Stanley Phillip Franklin and R. H. Sorgenfrey, Closed and image-closed

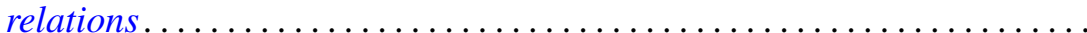

William Jesse Gray, A note on topological transformation groups with a

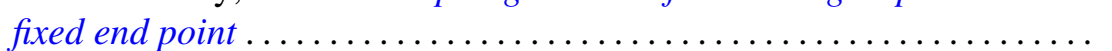

Myron Goldstein, $K$ - and L-kernels on an arbitrary Riemann surface ...... 449

George Joseph Kertz and Francis Regan, The exponential analogue of a generalized Weierstrass series .............................

Walter Leighton, On Liapunov functions with a single critical point ........ 467

Bernard Werner Levinger and Richard Steven Varga, On a problem of $O$.

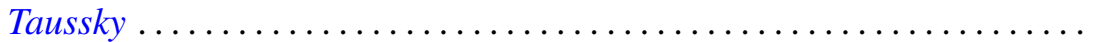

Lowell Duane Loveland, Tame subsets of spheres in $E^{3} \ldots \ldots \ldots \ldots \ldots . .489$

Erik Andrew Schreiner, Modular pairs in orthomodular lattices ......... 519

K. N. Srivastava, On dual series relations involving Laguerre polynomials ...............................

Arthur Steger, Diagonability of idempotent matrices.....

Walter Strauss, On continuity of functions with values in various Banach spaces...

Robert Vermes, On the zeros of a linear combination of polynomials ...

Elliot Carl Weinberg, On the scarcity of lattice-ordered matrix rings ....

Harold Widom, Toeplitz operators on $H_{p} \ldots \ldots \ldots \ldots \ldots$

Neal Zierler, On the lattice of closed subspaces of Hilbert space...

Irving Leonard Glicksberg, Correction to: "Maximal algebras and a theorem of Rado"

John Spurgeon Bradley, Correction to: "Adjoint quasi-differential operators of Euler type"

William Branham Jones, Erratum: "Duality and types of completeness in locally covex spaces".

Stanley P. Gudder, Erratum: "Uniqueness and existence properties of bounded observables" 